\title{
Atmospheric Breakdown Limitations to Optical Maser Propagation ${ }^{1,2}$
}

\author{
Richard G. Tomlinson
}

\author{
Antenna Laboratory, Department of Electrical Engineering, Ohio State University, Columbus, Ohio
}

(Received May 19, 1965; revised June 22, 1965)

\begin{abstract}
It is shown that air breakdown by focused optical maser pulses is dependent on gas pressure. It would appear that megawatt type pulses of 10 to 100 nanosecond duration could produce air breakdown.
\end{abstract}

The propagation of high-power optical maser beams is ultimately limited by the electrical breakdown of atmospheric gases. Data will be presented in this paper on breakdown induced in air by megawatt type pulses from mechanically $Q$-switched ruby and neodymium optical masers. With pulses of this type, breakdown exhibits a threshold. Below threshold little or no gas ionization is produced, but when the threshold conditions are exceeded large ionization rates lead to visible discharges and high-density plasmas. The occurrence of a visible discharge and the attendant abrupt attenuation of the transmitted optical maser energy will be referred to as breakdown.

An experimental arrangement for studying optical maser-induced gas breakdown is shown in figure 1 . The optical maser beam is brought to a focus inside a suitable chamber by the first lens and recollimated by the second lens. If no visible discharge occurs in the focal region, the optical maser beam passes through the chamber virtually unaffected. If, however, threshold conditions are exceeded, breakdown occurs at the focal region producing a plasma of such high density that further radiation is almost completely absorbed.

${ }^{1}$ The research in this paper was prepared under Contract AF 33(615)-2287 between the Antenna Laboratory, the Ohio State University Research Foundation, Columbus, Ohio, and the Air Force Avionics Laboratory, Research and Technology Division, Air Force Sysand the Air Force Avionics Laboratory, Research and Technology Division, Air Force

${ }_{2}$ This paper was presented at the Conference on Atmospheric Limitations to Optical Propagation, Boulder, Colo., March 18-19, 1965.

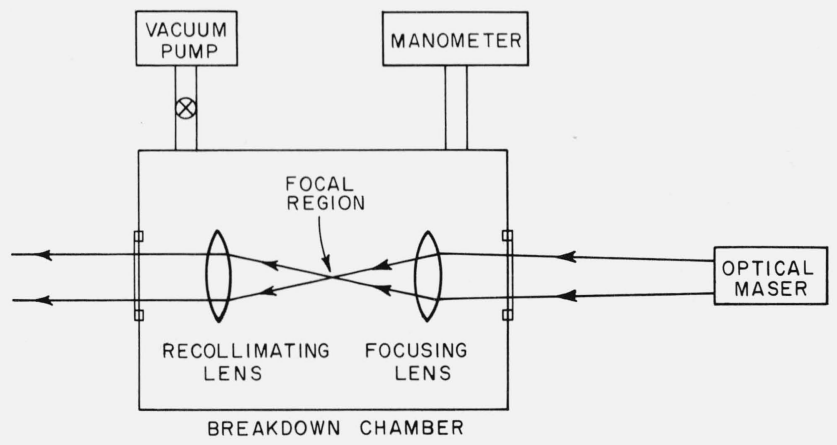

Figure 1. Breakdown chamber.
The transmitted pulses detected leaving the breakdown chamber are shown in figure 2. These traces were recorded by $1000-\mathrm{Mc} / \mathrm{s}$ oscilloscope and a fastresponse biplanar photodiode, with a net detection response time of the order of 1 nsec. The sharp breaks in these curves are due to the attenuation resulting when a plasma is formed in the focal region. Filtering the input to the photodiode established that the broadband radiation associated with the visible discharge began at approximately the same time as the onset of absorption in the transmitted energy. The data shown in figure 2 were taken in argon to illustrate the dependence of breakdown on pulse shape as well as optical power density. These data were selected from a large body of data to show a general correlation between the pulse shape and the time required to produce a breakdown. Deviations of \pm 5 nsec from this relationship were observed. The time interval until breakdown can be so long that the discharge occurs when the intensity in the focal region is only a fraction of what its peak value had been earlier when no discharge occurred. A similar effect, the discharge occurring later and later relative to the beginning of the pulse, is observed if, for a fixed incident pulse, the gas pressure is successively reduced. The breakdown in air exhibits grossly the same characteristics as breakdown in argon except that the dependence on pulse shape is more complex than that shown in figure 2 for argon. Breakdown data for air are also more erratic, apparently due to the complexity of the gas mixture. In general the slowest rising (although

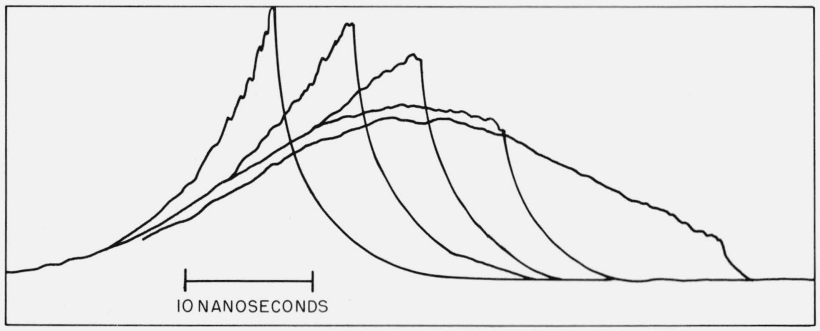

FIGURE 2. Transmitted optical maser pulses. 
not necessarily the smallest peak amplitude) pulse requires the longest time to produce air breakdown.

These observations are consistent with the assumptions that a small initial ionization is created early in the pulse, possibly by multiphoton absorption, and that this initial ionization grows in a cascade process to visible discharge densities. The mechanisms by which energy is transferred to the electron during the cascade process are presumably due to collisions with ions and neutral molecules in the presence of the optical maser field.

It should be noted that, for breakdown due to very high-power optical masers, quite different characteristics are to be expected, since ionization would be due primarily to multiphoton effects and not to a cascade growth process.

Figure 3 gives the peak power density transmitted versus the peak incident power density in air. Data are shown for a 3-cm focal-length lens and a $1.2-\mathrm{cm}$ focal-length lens, with measured focal areas of 4.4 $\times 10^{-5} \mathrm{~cm}^{2}$ and $6.6 \times 10^{-6} \mathrm{~cm}^{2}$, respectively, at halfintensity contours. For each lens two pressures, 400 torr and 1255 torr, are shown. The visible discharge occurred at higher power densities for the lower pressures because the plasma growth time was longer. The breakdown power density at a given pressure is not the same for the two lenses. This is presumably due to higher diffusion losses during the plasma growth process for the smaller spot size.

The experimental arrangement shown in figure 4 was used to change the focal spot size without changing lenses and to eliminate the dependence of the spot size on the beam divergence. A long focal-length lens imaged the optical maser beam on a tungsten aperture stop. A second lens, beyond the aperture, imaged the aperture inside a breakdown chamber. By varying the distance from the second lens to the aperture, the magnification ratio for the focused spot was changed.

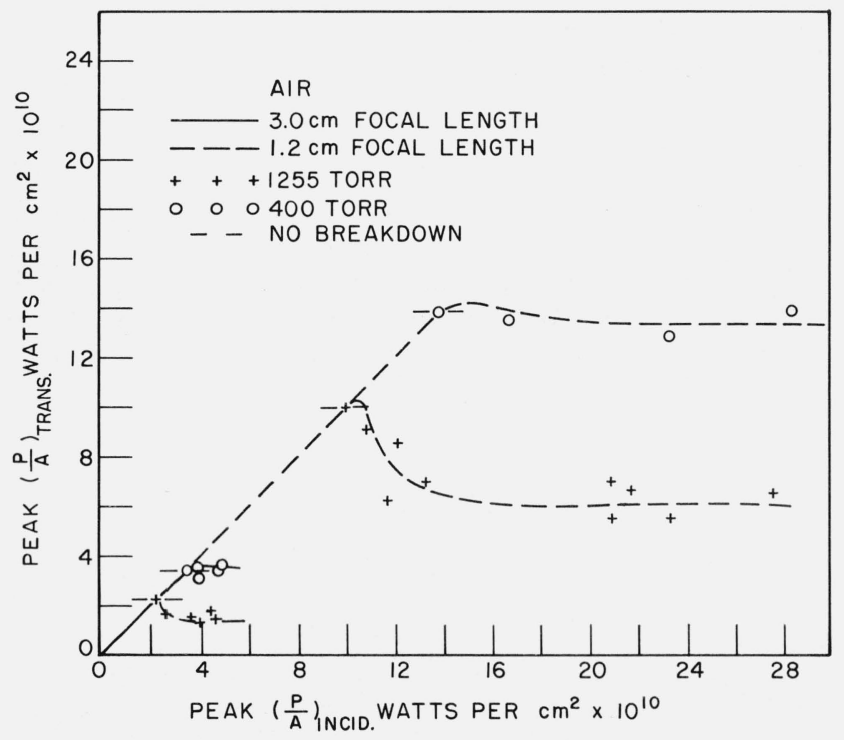

FIGURE 3. Peak power density transmitted versus peak power density incident.

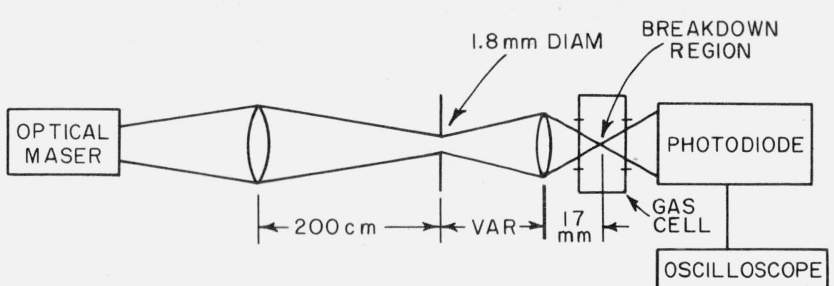

FigURE 4. Experimental arrangement for controlling focused spot size.

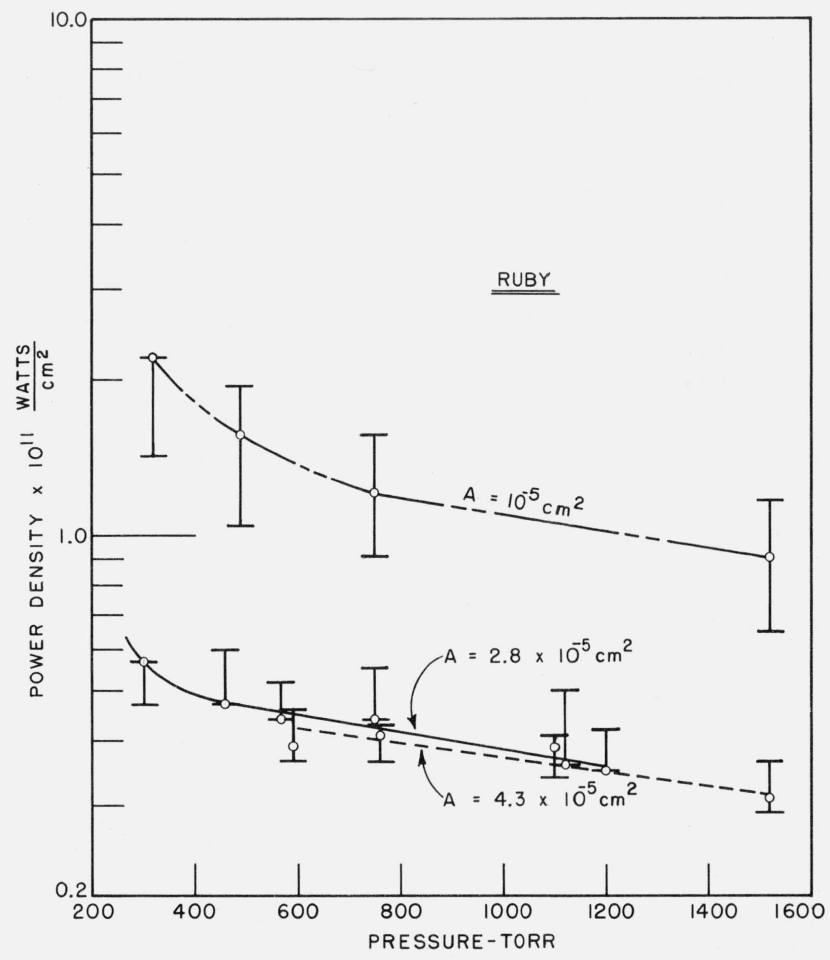

Figure 5. Peak power density of breakdown pulse for ruby optical maser.

Figure 5 shows the resulting breakdown density in air as a function of gas pressure for three different focused spot sizes of $10^{-5} \mathrm{~cm}^{2}, 2.8 \times 10^{-5} \mathrm{~cm}^{2}$, and $4.3 \times 10^{-5} \mathrm{~cm}^{2}$. These spot sizes were not measured but were calculated from geometrical optics. These data are in good agreement with those shown in figure 3. As before, higher diffusion losses made higher power densities necessary to produce breakdown for smaller focused spots. This diffusion limitation becomes particularly severe at low pressures. Over the pressure range investigated, the diffusion loss had become very small for the $4.3 \times 10^{-5} \mathrm{~cm}^{2}$ spot size, and one would expect the unfocused pulse to produce breakdown at about the same power densities had pulses of sufficient amplitude been available. It is not possible from these data to predict the breakdown power densities for longer pulses or CW sources, since we do not know the lowest optical power density which will still produce ionization.

The variations in peak power densities of pulses producing breakdown at a given pressure are indicated by error flags in figure 5 . These variations are due 


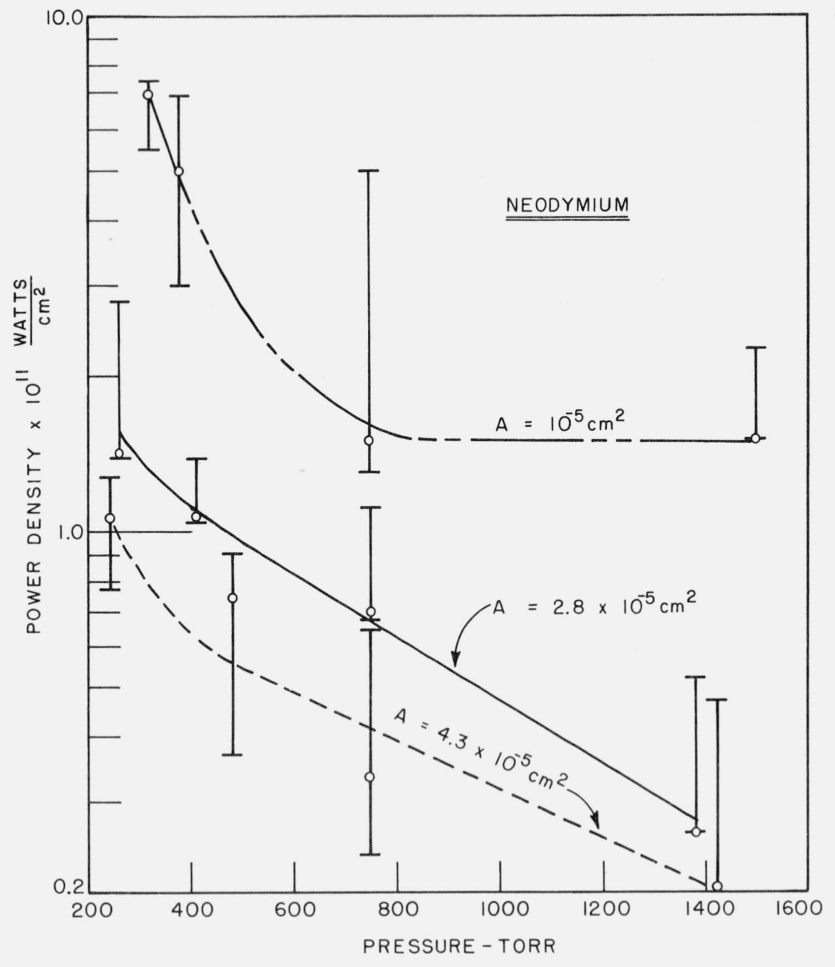

Figure 6. Peak power density of breakdown pulse for neodymium optical maser. both to different pulse shapes and to randomness in the breakdown process. In order to compare data for similar pulses, the peak power density of the pulse which required nearly the entire pulse width to produce breakdown is indicated for each pressure.

Figure 6 shows breakdown data for the same experimental arrangement using a neodymium-in-glass optical maser operating at $10,600 \AA$, rather than the ruby optical maser operating at $6943 \AA$. The behavior of these data seems to indicate a greater diffusion loss than did the ruby data. It is possible that the smaller beam divergence of the neodymium optical maser was sufficient to produce a focal spot smaller than the $1.8-\mathrm{mm}$ aperture shown in figure 4 so that the calculated spot sizes in figure 6 are too large.

This paper has shown that air breakdown by focused optical maser pulses is dependent on gas pressure, optical power density, focused spot size, and the temporal variation of optical power density. It appears that megawatt type pulses of $10^{-8}$ to $10^{-7}$ sec duration should produce air breakdown at optical power densities of about $10^{10} \mathrm{~W} / \mathrm{cm}^{2}$ if the crosssectional area of the optical maser beam is sufficiently large to produce negligible diffusion loss. These data do not presently allow predictions of breakdown power densitites for larger pulse durations or $\mathrm{CW}$ sources.

(Paper 69D11-576) 